ISSN 1980-5098

\title{
AGREGAÇÃO DE UM CAMBISSOLO HÚMICO EM POVOAMENTOS DE Pinus taeda L. COM DIFERENTES ROTAÇÕES
}

\author{
AGGREGATION OF A HAPLUMBREPT IN Pinus taeda L. STANDS WITH DIFFERENTS \\ ROTATIONS
}

\author{
Simone Filipini Abrão ${ }^{1}$ Suzana Ferreira da Rosa ${ }^{2}$ Dalvan José Reinert ${ }^{3}$
}

José Miguel Reichert ${ }^{3}$ Ângelo Augusto Ebling ${ }^{4}$

\begin{abstract}
RESUMO
O estudo tem como objetivo verificar se a introdução da espécie Pinus taeda L. em solo sob campo natural altera a agregação de um Cambissolo Húmico, em diferentes rotações. O estudo foi conduzido na região dos Campos de Cima da Serra, no município de Cambará do Sul - RS, Brasil. A fim de alcançar o objetivo acima descrito, foram selecionadas duas áreas de estudo, uma em primeira (RT1) e a outra em segunda rotação (RT2), com 13 anos de idade. Para cada povoamento, foi avaliada conjuntamente uma área de campo natural adjacente (testemunha). Em cada área, foram abertas cinco trincheiras e coletadas amostras de solo nas camadas 0,0-0,05, 0,05-0,20 e 0,20-0,40 m para a determinação da porcentagem de agregados estáveis em cada classe (AGRi) e do diâmetro médio geométrico de agregados estáveis em água (DMG). Não houve alteração da estabilidade de agregados na camada superficial $(0,0-0,05 \mathrm{~m})$. No entanto, nas camadas mais subsuperficiais $(0,05-0,20$ e 0,20-0,40 m), apenas a RT1 manteve a estabilidade de agregados maiores. Devido ao manejo adotado na RT2, como a queima e o corte raso, verificou-se a redução da estabilidade dos macroagregados e aumento dos agregados menores, ao passo que no campo natural a estabilidade em profundidade foi mantida.
\end{abstract}

Palavras-chave: estabilidade de agregados; florestamento; qualidade do solo.

\section{ABSTRACT}

The study aims to verify whether the introduction of the specie Pinus taeda L. in soil under natural field alters the aggregation of Haplumbrept, at differents rotations. The study was conducted in the region of Campos de Cima da Serra, in the municipality of Cambará do Sul - RS state, Brazil. In order to achieve the goal above described, were selected two study areas, one in the first rotation (RT1) and another in the second rotation (RT2), with 13 years of old. For each stand, a combined area of natural field adjacent (control) was assessed. In each area, five trenches were opened and soil samples collected at depths from 0.0 to 0.05 , 0.05 to 0.20 and 0.20 to $0.40 \mathrm{~m}$ to determine the percentage stable aggregates in each class (AGRI) and geometric mean diameter water stable aggregates (DMG). There was no change in the aggregate stability in the surface layer $(0.0$ to $0.05 \mathrm{~m})$. However, the subsurface layers $(0.05$ to 0.20 and 0.20 to $0.40 \mathrm{~m})$, just RT1 maintained stability of larger aggregates. Due to management adopted in RT2, such as burn and clear cut, there was a reduction in stability and increase in macro-aggregates smaller aggregates, whereas in the natural field stability in depth was maintained.

Keywords: aggregate stability; forestation; soil quality.

1 Engenheira Florestal, Doutoranda em Engenharia Florestal, Universidade Federal do Paraná, Av. Pref. Lothário Meissner, 900, Jardim Botânico, Campus III, CEP 80210-170, Curitiba (PR), Brasil. simone.abrao@ufpr.br

2 Engenheira Florestal, Dr ${ }^{\mathrm{a}}$., Professora do Instituto Federal de Educação, Ciência e Tecnologia do Rio Grande do Sul, Rua Nelsi de Ribas Fritsch, 1111, Esperança, CEP 98200-000, Ibirubá (RS), Brasil. suzanafdr@yahoo.com.br

3 Engenheiro Agrônomo, Dr., Professor do Departamento de Solos, Centro de Ciências Rurais, Universidade Federal de Santa Maria, Av. Roraima, 1000, CEP 97105-900, Santa Maria (RS), Brasil. dalvan@ufsm.br/ reichert@ufsm.br

4 Engenheiro Florestal, Dr., Professor do Curso de Engenharia Florestal, Universidade Federal Rural da Amazônia, Campus Parauapebas, Caixa Posta nº 3017, CEP 68515-970, Parauapebas (PA), Brasil. angelo.ebling@ufra.edu.br 


\section{INTRODUÇÃO}

As áreas com florestas plantadas no Brasil, em 2015, totalizaram 7.211.846 hectares com espécies de Eucalyptus spp e Pinus spp (INDÚSTRIA BRASILEIRA DE ÁRVORES, 2016). Segundo a mesma fonte, apesar da tendência de redução da área plantada em prol da substituição por plantios de eucalipto, estima-se que existam no país 1.581.239 hectares de áreas plantadas com espécies do gênero Pinus, sendo que $88,4 \%$ (1.398.171 ha) das áreas concentram-se na região Sul, estando o Rio Grande do Sul com 11,7\% desse percentual.

As espécies de pinus vêm sendo plantadas no Brasil há mais de um século pelos imigrantes europeus, tendo por objetivo a produção da madeira para o abastecimento industrial, processamento mecânico, produção de madeira serrada, madeira laminada, confecção de painéis ou na produção de celulose e papel. Aliado às práticas silviculturais adequadas, o pinus adaptou-se bem ao solo e ao clima, constituindo-se uma boa alternativa para suprir as demandas dos distintos setores industriais. Logo, a região localizada no extremo nordeste foi a que melhor desenvolveu a cultura do pinus no Rio Grande do Sul. No entanto, a substituição do campo natural por florestamentos tem sido objeto de estudos e discussões no que diz respeito aos impactos ambientais, principalmente na região dos Campos de Cima da Serra, na qual predominam os Cambissolos Húmicos.

Os Cambissolos são definidos como solos frágeis quanto aos aspectos morfogenéticos, considerados unidades pedológicas em plena transformação, com baixo grau de intemperismo, caracterizados por apresentar um horizonte B incipiente e elevados teores de silte ao longo do perfil. Os teores de silte em relação à argila podem promover o selamento superficial de algumas camadas, dificultando a infiltração de água e aumentando o escoamento superficial e a erosão no solo (RESENDE et al., 2014), fatores que tornam esses solos naturalmente suscetíveis à degradação. Nesse sentido, os agregados estáveis em água restabelecem a porosidade do solo influenciando no processo de infiltração e resistência à erosão (VICENTE et al., 2012); produzindo efeitos sobre a dinâmica da matéria orgânica e a ciclagem de nutrientes, confirmando a importância da estabilidade dos agregados para a manutenção da qualidade estrutural do solo e da produtividade dos sítios.

Muitos estudos relacionados à introdução do pinus têm inferido sobre as possíveis alterações na qualidade estrutural do solo. Autores como Martins et al. (2002), Shrestha et al. (2007) e Kato et al. (2010) têm estudado essa questão. Porém, torna-se difícil prever se os comportamentos descritos pelos trabalhos se mantêm ao longo do tempo, pois a grande maioria das pesquisas existentes relacionadas às alterações do solo não consideram seus efeitos nas sucessivas rotações, as quais podem refletir as condições da qualidade do solo de um determinado período.

Geralmente considera-se que o solo de campo natural apresenta características físicas, tal como a agregação, mais adequadas ao crescimento das plantas do que solos com outros usos. Este resultado, na maioria das vezes, é atribuído à alta densidade de raízes que contribuem para a aproximação das partículas do solo pela constante absorção de água do perfil do solo, às periódicas renovações do sistema radicular e à uniformidade de distribuição de exsudatos no solo, indicando, assim, que as gramíneas incrementam o teor de matéria orgânica e a estabilidade dos agregados.

Assim, mudanças no uso do solo, variações na atividade e natureza de micro-organismos, ação do sistema radicular (REICHERT et al., 2003) e a própria qualidade do material orgânico depositado, podem alterar de forma permanente ou temporária as condições de agregação e, por conseguinte, a estabilidade dos agregados (WOHLENBERG et al., 2004; BOCHNER et al., 2008). Portanto, a conversão do solo de campo natural em florestamento de pinus pode promover alterações nas propriedades do solo, em que o tempo de cultivo torna-se um fator importante, sendo necessários estudos que avaliem as condições da qualidade estrutural do solo durante os ciclos de rotações.

Diante do exposto, a estabilidade de agregados, como atributo físico, torna-se fundamental na avaliação da qualidade estrutural, visto que a mesma é sensível a variações no uso do solo, podendo identificar possíveis alterações promovidas pela conversão do campo natural em povoamentos de Pinus taeda ao longo das rotações. Assim, o objetivo deste estudo foi verificar o efeito da introdução da espécie Pinus taeda na agregação de um Cambissolo Húmico, em diferentes rotações. 


\section{MATERIAL E MÉTODOS}

\section{Caracterização dos locais de estudo e coleta de amostras de solo}

O estudo foi conduzido no município de Cambará do Sul - RS, em um Cambissolo Húmico Alumínico típico (EMBRAPA, 2013), com clima tipo Cfb1 (Köppen), utilizando povoamentos florestais em primeira e segunda rotação de Pinus taeda e em campo natural adjacente adotado como testemunha. A área de estudo de primeira rotação (RT1), com coordenadas geográficas de $29^{\circ} 06^{\prime}$ de latitude Sul e $50^{\circ} 11^{\prime}$ de longitude Oeste, possui 20,13 ha plantados com Pinus taeda (Figura 1a). O plantio foi realizado sem adubação, após marcação e coroamento manual. No campo natural, observou-se o predomínio de espécies rizomatosas do gênero Paspalum, formando um estrato rasteiro recobrindo todo o solo, o qual sustentava a criação de gado de forma extensiva.

A segunda área de estudo trata-se de uma área de reforma, ou seja, foi implantada onde já havia anteriormente o plantio com Pinus taeda e, portanto, de segunda rotação (RT2). Com coordenadas geográficas de $28^{\circ} 53^{\prime}$ de latitude Sul e 50 07' de longitude Oeste, abrange uma área de 636,14 ha florestados com a espécie Pinus taeda (Figura 1b). Nessa área, o corte final da primeira rotação foi executado de modo manual, com enleiramento dos resíduos. O plantio foi realizado sem adubação, após queima, marcação e coroamento manual. A área de segunda rotação vem sendo utilizada com florestamento de pinus há aproximadamente 30 anos.
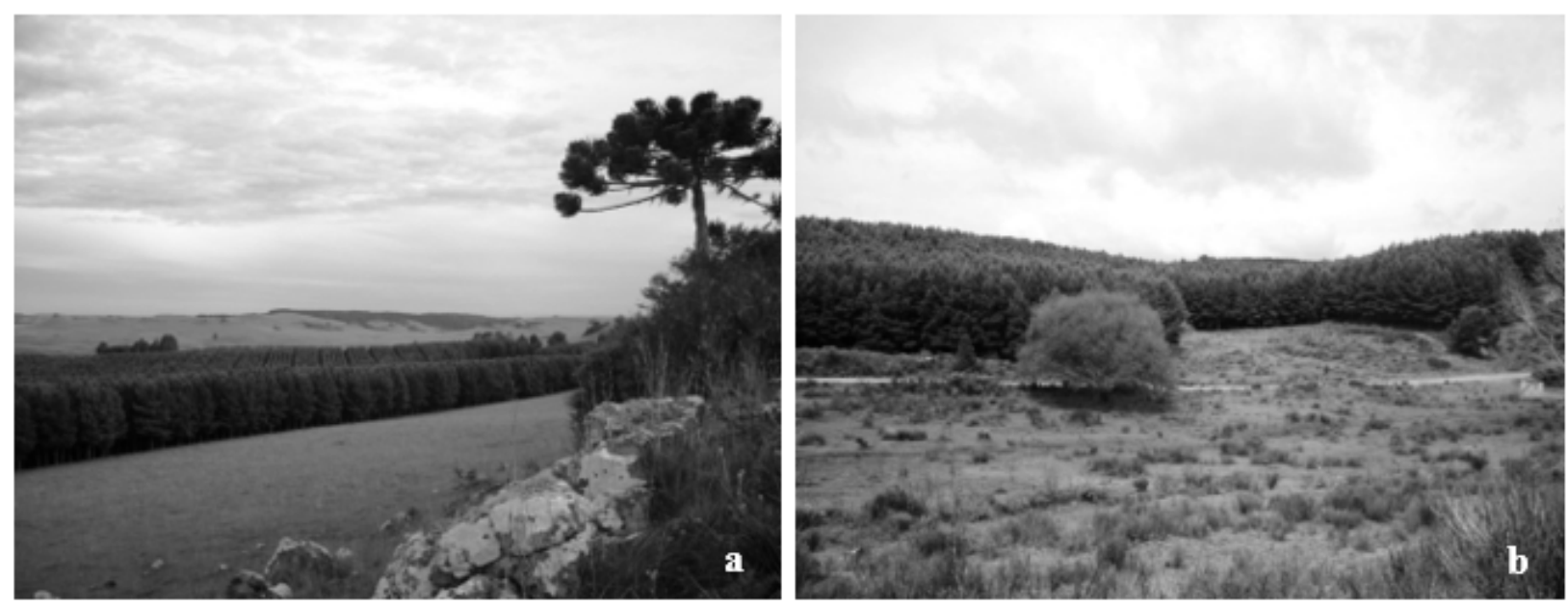

FIGURA 1: Área de campo natural e, ao fundo, povoamento de Pinus taeda em primeira (a) e segunda rotação (b), respectivamente, em Cambará do Sul - RS, Brasil.

FIGURE 1: Area of grassland and, to background, stand of Pinus taeda in first (a) and second rotation (b), respectively, in Cambará do Sul, RS state, Brazil.

Os dois povoamentos foram implantados em 1996, em espaçamento 3 x 2 m, com população de 1.666 mudas por hectare. Ambos os povoamentos possuem a mesma idade (13 anos), porém em ciclos de rotações distintos. Além disso, antes do florestamento, as duas áreas de estudo encontravam-se sob campo natural, favorecendo a avaliação da qualidade estrutural do solo após a introdução da espécie Pinus taeda.

A coleta das amostras em cada área de estudo foi realizada em março de 2010, por meio da abertura de trincheiras nas entrelinhas dos plantios da RT1 e RT2 e nas suas respectivas testemunhas: CN1 e CN2 (áreas de campo natural adjacentes). Desse modo, foram abertas cinco trincheiras em cada área de estudo, totalizando 20 pontos de coleta. Em cada ponto, no centro de cada camada $(0,00-0,05 \mathrm{~m}, 0,05-0,20 \mathrm{~m}$ e 0,20-0,40 m de profundidade), coletaram-se amostras com estrutura alterada e preservada, esta última, em cilindros metálicos com $0,03 \mathrm{~m}$ de altura e $0,06 \mathrm{~m}$ de diâmetro. 


\section{Análises físicas do solo}

Realizou-se a análise granulométrica por meio da quantificação do teor de argila $(<0,002 \mathrm{~mm})$, silte $(0,002-0,05 \mathrm{~mm})$, areia fina $(0,05-0,20 \mathrm{~mm})$ e areia grossa $(>0,20 \mathrm{~mm})$ pelo método da pipeta (EMBRAPA, 2011) e adaptado ao Laboratório de Física do Solo da Universidade Federal de Santa Maria. Para isso, foram utilizados $20 \mathrm{~g}$ de terra fina seca ao ar, $50 \mathrm{ml}$ de água destilada e $10 \mathrm{ml}$ de hidróxido de sódio (NaOH 6\%) como dispersante químico. As amostras foram acondicionadas e agitadas por cinco minutos em agitador horizontal, mantidas em repouso durante a noite, e agitadas por mais quatro horas. Os dados da análise granulométrica permitiram verificar que as duas áreas de estudo apresentaram textura argilosa nas camadas avaliadas (Tabela 1).

A densidade do solo (Ds) foi determinada pelo método do cilindro (EMBRAPA, 2011). A porosidade total do solo $(\mathrm{Pt})$ foi calculada indiretamente a partir da relação entre a densidade do solo e a densidade de partículas (Dp), sendo a Dp obtida pelo método do balão volumétrico modificado de acordo com a metodologia proposta por Gubiani, Reinert e Reichert (2006). O grau de compactação (Gc) foi calculado conforme a equação:

$$
\mathrm{Gc}=\frac{\mathrm{Ds}}{\mathrm{Ds}_{\mathrm{IHO}}} \times 100
$$

Em que: $\mathrm{Gc}=$ grau de compactação $(\%)$; $\mathrm{Ds}=$ densidade do solo $\left(\mathrm{Mg} \mathrm{m}^{-3}\right)$ e $\mathrm{Ds}_{\mathrm{HH}}$ : densidade crítica estabelecida a partir do intervalo hídrico ótimo $\left(\mathrm{Ds}_{\mathrm{IHO}}=-0,00078 \mathrm{x} \%\right.$ argila $+1,83803$, conforme Reichert et al. (2009).

O intervalo hídrico ótimo (IHO) é um índice baseado na densidade do solo, que pondera a faixa

TABELA 1: Frações granulométricas e suas respectivas classes texturais de um Cambissolo Húmico, para as áreas e camadas em estudo, em Cambará do Sul - RS, Brasil.

TABLE 1: Size fractions and their textural classes of a Haplumbrept, for the areas and layers under study, in Cambará do Sul, RS state, Brazil.

\begin{tabular}{|c|c|c|c|c|c|c|}
\hline \multicolumn{6}{|c|}{ Areia } & \multirow{3}{*}{ Classe Textural } \\
\hline Camada & Total & Grossa & Fina & Silte & Argila & \\
\hline \multirow{2}{*}{\multicolumn{7}{|c|}{ Área de $1^{\text {a }}$ rotação de Pinus taeda }} \\
\hline & & & & & & \\
\hline $0,00-0,05$ & 156 & 102 & 54 & 275 & 569 & Argilosa \\
\hline $0,05-0,20$ & 165 & 106 & 59 & 297 & 538 & Argilosa \\
\hline $0,20-0,40$ & 179 & 125 & 54 & 299 & 522 & Argilosa \\
\hline \multicolumn{7}{|c|}{ Campo natural adjacente a $1^{\mathrm{a}}$ rotação } \\
\hline $0,00-0,05$ & 179 & 116 & 63 & 322 & 499 & Argilosa \\
\hline $0,05-0,20$ & 162 & 108 & 54 & 342 & 496 & Argilosa \\
\hline $0,20-0,40$ & 157 & 105 & 52 & 255 & 588 & Argilosa \\
\hline \multicolumn{7}{|c|}{ Área de $2^{\mathrm{a}}$ rotação de Pinus taeda } \\
\hline $0,00-0,05$ & 208 & 123 & 85 & 302 & 490 & Argilosa \\
\hline $0,05-0,20$ & 215 & 135 & 80 & 297 & 488 & Argilosa \\
\hline $0,20-0,40$ & 195 & 126 & 69 & 240 & 565 & Argilosa \\
\hline \multicolumn{7}{|c|}{ Campo natural adjacente a $2^{\mathrm{a}}$ rotação } \\
\hline $0,00-0,05$ & 175 & 104 & 71 & 308 & 517 & Argilosa \\
\hline $0,05-0,20$ & 170 & 106 & 64 & 329 & 501 & Argilosa \\
\hline $0,20-0,40$ & 192 & 124 & 68 & 263 & 545 & Argilosa \\
\hline
\end{tabular}


de umidade do solo no qual se espera que não ocorram limitações para o crescimento da planta quando se considera a aeração do solo, resistência à penetração e água disponível para as plantas (REICHERT et al., 2009). Os autores verificaram uma relação direta entre o teor de argila e a densidade crítica obtida pelo IHO, conforme a equação descrita acima. Para obter o IHO, consideraram como valores críticos o teor de água na capacidade de campo (tensão matricial de $0,01 \mathrm{MPa}$ ), ponto de murcha permanente (tensão matricial de 1,5 $\mathrm{MPa}$ ), teor de água quando a resistência à penetração é igual a $2 \mathrm{MPa}$ e o teor de água quando a porosidade de aeração é de $0,10 \mathrm{~m}^{3} \mathrm{~m}^{-3}$. A Ds $\mathrm{IHO}_{\mathrm{IHO}}$ foi considerada como a densidade onde o IHO é zero.

Para a determinação da distribuição do tamanho dos agregados estáveis em água, as amostras com estrutura preservada foram secas à sombra e destorroadas manualmente, através de seus planos de fraqueza localizados na junção interagregados, de modo que toda a massa de solo atravessasse a peneira com malha de $8,0 \mathrm{~mm}$.

A distribuição do tamanho dos agregados foi obtida pela metodologia modificada de Kemper e Chepil (1965), na qual os agregados são submersos por 10 minutos para umedecimento e, posteriormente, agitados no aparelho de oscilação vertical de Yoder (1936), em um conjunto de peneiras com malhas de 4,$75 ; 2,00 ; 1,00$ e $0,21 \mathrm{~mm}$ em seu interior. $\mathrm{O}$ aparelho foi preparado para manter 30 oscilações por minuto, de maneira que os agregados fossem peneirados em imersão por mais 10 minutos, separando-os em cinco classes de tamanho: 8,00-4,75; 4,75-2,00; 2,00-1,00; 1,00-0,21 mm e $<0,21 \mathrm{~mm}$.

Os resultados foram expressos pela porcentagem de agregados de cada classe (AGRi) e diâmetro médio geométrico de agregados estáveis em água (DMG), calculados por meio das equações:

$$
\begin{aligned}
& \text { AGRi }=m A G R i ~ \sum_{i=1}^{n} \text { AGRi } \\
& \text { DMG }=\operatorname{EXP} \mid \sum_{i=1}^{n}\left(\text { AGRi.ln(ci)) } \sum_{i=1}^{n} \text { AGRi }\right]
\end{aligned}
$$

Em que: $\mathrm{AGRi}=$ agregados obtidos em cada classe (\%); $\mathrm{mAGR}_{\mathrm{i}}=$ massa de agregados da classe $\mathrm{i}$ (menos a massa de areias e outros materiais inertes) $(\mathrm{g}) ; \sum_{\mathrm{i}=1}^{\mathrm{n}} \mathrm{AGRj}=$ massa total de agregados (menos areias e outros materiais inertes) $(\mathrm{g})$ e $\ln (\mathrm{ci})=$ logaritmo natural das classes de diâmetro das peneiras.

\section{Análises químicas do solo}

Analisaram-se os atributos químicos do solo carbono orgânico total (COT), alumínio (Al), cálcio $(\mathrm{Ca})$, magnésio $(\mathrm{Mg})$ e potássio $(\mathrm{K})$ trocáveis, fósforo disponível $(\mathrm{P}), \mathrm{pH}$ em água e $\mathrm{pH}_{\mathrm{SMP}}$, conforme a metodologia descrita em Tedesco et al. (1995).

O COT foi determinado por oxidação via seca em analisador elementar. O Al foi obtido através da titulação de $\mathrm{NaOH}(0,0116 \mathrm{~N})$ em $\mathrm{KCl} 1 \mathrm{M}$. O Ca e o Mg foram extraídos em $\mathrm{KCl} 1 \mathrm{M}$ e determinados em espectrofotômetro de absorção atômica. O K e o P foram obtidos pelo extrator Mehlich, sendo as leituras realizadas em espectrofotômetro de colorimetria e fotômetro de chamas, respectivamente. $\mathrm{O}$ pH em água na proporção 1:1 e o $\mathrm{pH}_{\mathrm{SMP}}$ foram quantificados através de pHmetro.

\section{Análise estatística}

Os dados encontrados para porcentagem de agregados de cada classe e diâmetro médio geométrico de agregados estáveis em água referentes à RT1 foram comparados com a sua testemunha, o CN1. Do mesmo modo, os dados relativos à RT2 foram comparados com a sua respectiva testemunha, o CN2. Para fins de comparação de médias, foi aplicado o teste " $\mathrm{t}$ " de Student ao nível de $5 \%$ de probabilidade de erro. Complementarmente foi realizada a análise de correlação de Spearman, na primeira (RT1 e CN1) e na segunda área de estudo (RT2 e CN2), entre a AGRi e o DMG de agregados estáveis em água com atributos físicos e químicos do solo. Para isso, consideraram-se as correlações que apresentaram significância pela tabela " $t$ ", sendo significantes e altamente significantes os resultados com probabilidades de $95 \%$ e $99 \%$ de confiança, respectivamente. 


\section{RESULTADOS E DISCUSSÃO}

Comparando a RT1 com o CN1, na camada de 0,00 a 0,05 m, os valores de distribuição do tamanho dos agregados estáveis em água não diferiram estatisticamente entre os usos do solo, exceto na classe de tamanho $<0,21 \mathrm{~mm}$, em que houve a maior formação significativa de agregados no pinus (Tabela 2). Valores elevados de agregados estáveis pertencentes às primeiras classes $(8,00$ a 4,75 e 4,75 a 2,00 mm) foram observados. Nestas, as médias na área com plantio de pinus foram de $46,26 \%$ e $28,27 \%$, respectivamente, demonstrando alta estabilidade de agregados com diâmetros maiores na camada superior do solo.

Na camada superficial do solo ( 0,00 a $0,05 \mathrm{~m})$, a RT1 manteve o mesmo comportamento do CN1 quanto ao percentual de agregados estáveis e ao DMG (Tabela 2). Isso pode ser decorrente ao fato do material orgânico permanecer acumulado na superfície do solo. Dessa forma, é natural que ambas as áreas apresentem altos percentuais de agregados com diâmetros maiores e, consequentemente, mais estáveis. Segundo Tisdall e Oades (1982), a matéria orgânica do solo é um dos principais agentes de formação e estabilização dos agregados, pois polímeros orgânicos ligam-se às superfícies inorgânicas por meio de cátions polivalentes e polímeros hidroximetálicos. A exemplo disso, observou-se uma correlação positiva

TABELA 2: Agregados estáveis em água em diferentes classes, diâmetro médio geométrico, carbono orgânico total, densidade do solo, porosidade total e grau de compactação de um Cambissolo Húmico, para as áreas e camadas em estudo, em Cambará do Sul - RS, Brasil.

TABLE 2: Water stable aggregates in different classes, geometric mean diameter, total organic carbon, bulk density, porosity and degree of compaction of a Haplumbrept, for the areas and layers under study, in Cambará do Sul, RS state, Brazil.

\begin{tabular}{|c|c|c|c|c|c|c|c|c|c|c|}
\hline \multicolumn{6}{|c|}{ Classes de Agregados (mm) } & \multirow{2}{*}{$\begin{array}{l}\mathrm{DMG} \\
(\mathrm{mm})\end{array}$} & \multirow{2}{*}{$\frac{\mathrm{COT}}{\left(\mathrm{g} \mathrm{kg}^{-1}\right)}$} & \multirow{2}{*}{$\frac{\text { Ds }}{\left(\mathrm{Mg} \mathrm{m}^{-3}\right)}$} & \multirow{2}{*}{$\frac{\mathrm{Pt}}{\left(\mathrm{m}^{3} \mathrm{~m}^{-3}\right)}$} & \multirow{2}{*}{$\begin{array}{l}\text { Gc } \\
(\%)\end{array}$} \\
\hline & $8,00-4,75$ & $4,75-2,00$ & $2,00-1,00$ & $1,00-0,21$ & $<0,21$ & & & & & \\
\hline \multicolumn{11}{|c|}{ Área de $1^{\mathrm{a}}$ rotação (RT1) e campo natural adjacente $(\mathrm{CN} 1)$} \\
\hline \multicolumn{11}{|c|}{ Camada $0,00-0,05 \mathrm{~m}$} \\
\hline RT1 & $46,26 \mathrm{a}$ & $28,27 \mathrm{a}$ & $9,80 \mathrm{a}$ & $10,80 \mathrm{a}$ & $4,87 \mathrm{~b}$ & $3,21 \mathrm{a}$ & $39,1 \mathrm{~b}$ & $0,89 \mathrm{a}$ & $0,64 \mathrm{a}$ & $49,72 \mathrm{a}$ \\
\hline $\mathrm{CN} 1$ & $53,82 \mathrm{a}$ & $22,59 \mathrm{a}$ & $9,85 \mathrm{a}$ & $10,95 \mathrm{a}$ & $2,79 \mathrm{a}$ & $3,40 \mathrm{a}$ & $49,9 \mathrm{a}$ & $0,88 \mathrm{a}$ & $0,63 \mathrm{a}$ & $48,88 \mathrm{a}$ \\
\hline \multicolumn{11}{|c|}{ Camada $0,05-0,20 \mathrm{~m}$} \\
\hline RT1 & $66,01 \mathrm{a}$ & $20,38 \mathrm{~b}$ & $6,29 \mathrm{~b}$ & $5,40 \mathrm{~b}$ & $1,92 \mathrm{a}$ & $4,18 \mathrm{a}$ & $37,6 \mathrm{a}$ & $1,00 \mathrm{a}$ & $0,59 \mathrm{a}$ & $55,55 \mathrm{a}$ \\
\hline $\mathrm{CN} 1$ & $52,50 \mathrm{~b}$ & $21,59 \mathrm{a}$ & $11,20 \mathrm{a}$ & $12,23 \mathrm{a}$ & $2,48 \mathrm{a}$ & $3,19 \mathrm{~b}$ & $37,6 \mathrm{a}$ & $1,00 \mathrm{a}$ & $0,60 \mathrm{a}$ & $55,55 \mathrm{a}$ \\
\hline \multicolumn{11}{|c|}{ Camada $0,20-0,40 \mathrm{~m}$} \\
\hline RT1 & $21,37 \mathrm{a}$ & $23,60 \mathrm{a}$ & $26,27 \mathrm{a}$ & $24,05 \mathrm{~b}$ & $4,70 \mathrm{a}$ & $1,79 \mathrm{a}$ & $26,7 \mathrm{a}$ & $1,08 \mathrm{a}$ & $0,58 \mathrm{a}$ & $60,00 \mathrm{a}$ \\
\hline $\mathrm{CN} 1$ & $15,26 \mathrm{a}$ & $17,49 \mathrm{~b}$ & $24,32 \mathrm{a}$ & $37,84 \mathrm{a}$ & $5,08 \mathrm{a}$ & $1,37 \mathrm{a}$ & $23,1 \mathrm{~b}$ & $1,07 \mathrm{a}$ & $0,58 \mathrm{a}$ & $59,78 \mathrm{a}$ \\
\hline \multicolumn{11}{|c|}{ Área de $2^{\mathrm{a}}$ rotação (RT2) e campo natural adjacente $(\mathrm{CN} 2)$} \\
\hline \multicolumn{11}{|c|}{ Camada $0,00-0,05 \mathrm{~m}$} \\
\hline RT2 & $43,35 \mathrm{a}$ & $26,15 \mathrm{a}$ & $13,19 \mathrm{a}$ & $13,18 \mathrm{a}$ & $4,13 \mathrm{a}$ & $2,83 \mathrm{a}$ & $47,6 \mathrm{a}$ & $0,84 \mathrm{a}$ & $0,65 \mathrm{a}$ & $46,67 \mathrm{a}$ \\
\hline $\mathrm{CN} 2$ & $38,87 \mathrm{a}$ & $24,08 \mathrm{a}$ & $14,71 \mathrm{a}$ & $18,36 \mathrm{a}$ & $3,98 \mathrm{a}$ & $2,52 \mathrm{a}$ & $40,6 \mathrm{a}$ & $0,96 \mathrm{a}$ & $0,61 \mathrm{a}$ & $53,33 \mathrm{a}$ \\
\hline \multicolumn{11}{|c|}{ Camada $0,05-0,20 \mathrm{~m}$} \\
\hline RT2 & $16,76 \mathrm{~b}$ & $26,43 \mathrm{a}$ & $26,77 \mathrm{a}$ & $24,34 \mathrm{a}$ & $5,70 \mathrm{a}$ & $1,54 \mathrm{~b}$ & $27,2 \mathrm{~b}$ & $1,04 \mathrm{a}$ & $0,58 \mathrm{a}$ & $57,78 \mathrm{a}$ \\
\hline $\mathrm{CN} 2$ & 36,17 a & $28,60 \mathrm{a}$ & $17,72 \mathrm{~b}$ & $14,70 \mathrm{~b}$ & $2,80 \mathrm{a}$ & $2,66 \mathrm{a}$ & $32,1 \mathrm{a}$ & $1,00 \mathrm{a}$ & $0,60 \mathrm{a}$ & $55,55 \mathrm{a}$ \\
\hline \multicolumn{11}{|c|}{ Camada $0,20-0,40 \mathrm{~m}$} \\
\hline RT2 & $1,66 \mathrm{~b}$ & $9,05 \mathrm{~b}$ & $24,33 \mathrm{a}$ & $54,59 \mathrm{a}$ & $10,36 \mathrm{a}$ & $0,78 \mathrm{~b}$ & $27,9 \mathrm{a}$ & $1,01 \mathrm{a}$ & $0,60 \mathrm{a}$ & $56,42 \mathrm{a}$ \\
\hline $\mathrm{CN} 2$ & $15,42 \mathrm{a}$ & $27,57 \mathrm{a}$ & $26,37 \mathrm{a}$ & $26,65 \mathrm{~b}$ & $3,99 \mathrm{~b}$ & $1,70 \mathrm{a}$ & $23,6 \mathrm{a}$ & $1,08 \mathrm{a}$ & $0,58 \mathrm{a}$ & $60,33 \mathrm{a}$ \\
\hline
\end{tabular}

Em que: Médias seguidas pela mesma letra na coluna, não diferem entre si pelo teste "t" a $95 \%$ de probabilidade. $\mathrm{RT} 1=$ rotação $1 ; \mathrm{RT} 2=$ rotação $2 . \mathrm{CN} 1=$ testemunha $1 ; \mathrm{CN} 2=$ testemunha 2. 
e altamente significativa entre o DMG e o COT $(\rho=0,62)$ e entre os agregados estáveis pertencentes à classe de 8,00 a $4,75 \mathrm{~mm}$ e o COT $(\rho=0,77)$, conforme descrito na Tabela 3. Ainda que o CN1 apresentasse maior teor de COT nessa camada $\left(49,9 \mathrm{~g} \mathrm{~kg}^{-1}\right)$, a RT1 $\left(39,1 \mathrm{~g} \mathrm{~kg}^{-1}\right)$ conseguiu promover agregação de forma similar, devido ao longo período de rotação sem interferência do manejo na área. Wendling et al. (2012) também perceberam maior teor de COT em áreas cultivadas (pastagem e semeadura direta), na camada superficial $(0,0$ a $0,10 \mathrm{~m})$. Mesmo assim, foram as áreas de cerrado, seguido de Pinus caribaea que apresentaram o maior DMG e diâmetro médio ponderado (DMP) de agregados estavéis em água. Este resultado demonstrou que em áreas pouco revolvidas, o tamanho dos agregados é maior.

Outrossim, os agregados estáveis pertencentes à classe de 8,00 a $4,75 \mathrm{~mm}$ tiveram correlação significativa com o silte $(\rho=0,43), \mathrm{Ca}(\rho=0,42), \mathrm{pH}$ em água e a CTC em $\mathrm{pH} 7(\rho=0,44)$; e altamente significativa com a Ds $(\rho=-0,61)$, Pt $(\rho=0,50)$, Gc $(\rho=-0,62), \operatorname{Mg}(\rho=0,54)$, P $(\rho=0,53)$ e K $(\rho=0,59)$. Dentre os citados, com exceção do silte, $\mathrm{Ca}$ e do $\mathrm{pH}$ em água, os demais atributos também se correlacionaram com o DMG. Nas menores classes (2,00-1,00 e 1,00-0,21 mm), observou-se correlação negativa e significativa com os teores de COT, $\mathrm{Mg}, \mathrm{K}, \mathrm{P}, \mathrm{pH}$ em água e CTC em $\mathrm{pH}$ 7. Na CTC dos solos, a matéria orgânica contribui no sentido de favorecer a retenção de cátions e diminuir as perdas por lixiviação. Esse aspecto é comprovado por meio da correlação com os cátions trocáveis. A própria composição da matéria orgânica, formada por moléculas grandes e amorfas (elevada área superficial específica), contribuem para elevar a CTC no solo e aproximar as partículas na formação dos agregados. Porém, à medida que as forças de atração entre os coloides diminuem, pode ocorrer a formação de agregados menores, bem como a redução dos fluxos de água e ar no solo, do diâmetro e volume dos poros, seja pela diminuição do teor da

TABELA 3: Coeficientes de correlação de Spearman entre o diâmetro médio geométrico, porcentagem de agregados em cada classe e atributos físicos e químicos encontrados na primeira área de estudo.

TABLE 3: Spearman's correlation coefficients between the geometric mean diameter, percentage of aggregates in each class and physical and chemical attributes found in the first area of study.

\begin{tabular}{lcccccc}
\hline \multirow{2}{*}{\multicolumn{1}{c}{ Atributo do solo }} & \multicolumn{5}{c}{ Classes de Agregados (mm) } \\
\cline { 2 - 6 } & $\begin{array}{c}\text { DMG } \\
(\mathrm{mm})\end{array}$ & $8,00-4,75$ & $4,75-2,00$ & $2,00-1,00$ & $1,00-0,21$ & $<0,21$ \\
\hline Areia total $\left(\mathrm{g} \mathrm{kg}^{-1}\right)$ & 0,06 & 0,03 & 0,13 & 0,19 & $-0,01$ & $-0,15$ \\
Silte $\left(\mathrm{g} \mathrm{kg}^{-1}\right)$ & 0,22 & $0,43^{*}$ & 0,02 & $-0,19$ & $-0,22$ & $-0,19$ \\
Argila $\left(\mathrm{g} \mathrm{kg}^{-1}\right)$ & $-0,15$ & $-0,30$ & 0,00 & 0,08 & 0,12 & 0,18 \\
Densidade do solo $\left(\mathrm{Mg} \mathrm{m}^{-3}\right)$ & $-0,58^{* *}$ & $-0,61^{* *}$ & $-0,48^{* *}$ & 0,34 & $0,50^{* *}$ & $0,40^{*}$ \\
Porosidade total $\left(\mathrm{m}^{3} \mathrm{~m}^{-3}\right)$ & $0,50^{* *}$ & $0,50^{* *}$ & $0,56^{* *}$ & $-0,16$ & $-0,37^{*}$ & $-0,40^{*}$ \\
Grau de compactação $(\%)$ & $-0,59^{* *}$ & $-0,62^{* *}$ & $-0,48^{* *}$ & 0,34 & $0,50^{* *}$ & $0,42^{*}$ \\
Carbono orgânico total $\left(\mathrm{g} \mathrm{kg}^{-1}\right)$ & $0,62^{* *}$ & $0,77^{* *}$ & 0,31 & $-0,52^{* *}$ & $-0,67^{* *}$ & $-0,49^{* *}$ \\
Al $\left(\mathrm{cmol} \mathrm{dm}^{-3}\right)$ & $-0,11$ & $-0,14$ & 0,01 & $-0,23$ & $-0,27$ & 0,24 \\
Ca $\left(\mathrm{cmol} \mathrm{dm}^{-3}\right)$ & 0,33 & $0,42^{*}$ & 0,10 & $-0,27$ & $-0,30$ & $-0,32$ \\
Mg $\left(\mathrm{cmol} \mathrm{dm}^{-3}\right)$ & $0,49^{* *}$ & $0,54^{* *}$ & $0,39^{*}$ & $-0,41^{*}$ & $-0,55^{* *}$ & $-0,34$ \\
$\mathrm{~K}\left(\mathrm{mg} \mathrm{dm}^{-3}\right)$ & $0,50^{* *}$ & $0,59^{* *}$ & $0,37^{*}$ & $-0,53^{* *}$ & $-0,62^{* *}$ & $-0,27$ \\
P $\left(\mathrm{mg} \mathrm{dm}^{-3}\right)$ & $0,46^{* *}$ & $0,53^{* *}$ & $0,46^{*}$ & $-0,50^{* *}$ & $-0,64^{* *}$ & $-0,27$ \\
pH em água & $-0,25$ & $-0,45^{*}$ & $-0,13$ & $0,62^{* *}$ & $0,75^{* *}$ & 0,00 \\
pH ${ }_{\text {sMP }}$ & $-0,23$ & $-0,30$ & $-0,15$ & $0,45^{*}$ & $0,57^{* *}$ & $-0,02$ \\
CTC em pH do solo $\left(\mathrm{cmol} \mathrm{dm}^{-3}\right)$ & 0,20 & 0,18 & 0,20 & $-0,34$ & $-0,44^{*}$ & $-0,05$ \\
CTC em pH 7 (cmol dm dm $\left.^{-3}\right)$ & $0,37^{*}$ & $0,44^{*}$ & 0,26 & $-0,52^{*}$ & $-0,65^{*}$ & $-0,13$ \\
\hline
\end{tabular}

Em que: $\mathrm{DMG}=$ diâmetro médio geométrico; $\mathrm{CTC}=$ capacidade de troca de cátions; $*$ significativo a $5 \%$ de probabilidade; $* *$ significativo a $1 \%$. 
matéria orgânica ou pelo adensamento natural proporcionado pelas camadas sobrejacentes, evidenciado, sobretudo, na correlação com a Ds, Pt e o Gc. Ressalva-se que a hidrofobicidade em solos sob pinus também pode favorecer a elevada estabilidade de agregados (BUCZKO; BENS; HÜTTL, 2006).

Por outro lado, a Ds, Pt e o Gc não diferiram significativamente entre as áreas de estudo. Os menores valores de Ds foram encontrados na camada de 0,00 a $0,05 \mathrm{~m}\left(0,89 \mathrm{Mg} \mathrm{m}^{-3}\right.$ e $\left.0,88 \mathrm{Mg} \mathrm{m}^{-3}\right)$ e maiores na camada de 0,20 a $0,40 \mathrm{~m}\left(1,08 \mathrm{Mg} \mathrm{m}^{-3}\right.$ e $\left.1,07 \mathrm{Mg} \mathrm{m}^{-3}\right)$, para RT1 e CN1, respectivamente. Os baixos valores de Ds ocorrem devido ao elevado teor de matéria orgânica presente no solo e à textura ser do tipo argilosa, o que confere maior agregação e porosidade total ao solo. Os maiores valores de Pt foram encontrados na camada de 0,00 a $0,05 \mathrm{~m}\left(0,64 \mathrm{~m}^{3} \mathrm{~m}^{-3}\right.$ e $\left.0,63 \mathrm{~m}^{3} \mathrm{~m}^{-3}\right)$ e menores na camada de 0,20 a $0,40 \mathrm{~m}\left(0,58 \mathrm{~m}^{3} \mathrm{~m}^{-3} \mathrm{e}\right.$ $0,58 \mathrm{~m}^{3} \mathrm{~m}^{-3}$ ), para RT1 e CN1, respectivamente. Para o Gc foram observados percentuais abaixo de 70\%, nesse caso, conclui-se que na maior Ds observada $\left(1,16 \mathrm{Mg} \mathrm{m}^{-3}\right)$ poderia ser acrescida em mais de $40 \%$ para se aproximar da densidade crítica $\left(1,79 \mathrm{Mg} \mathrm{m}^{-3}\right)$ estabelecida a partir do intervalo hídrico ótimo ( $\mathrm{Ds}_{\mathrm{IHO}}$ ), proposta por Reichert et al. (2009).

Na segunda camada avaliada $(0,05$ a $0,20 \mathrm{~m})$, a RT1 diferenciou-se estatisticamente do CN1 em todas as classes de agregados, com exceção da classe $<0,21 \mathrm{~mm}$ (Tabela 2). A estabilidade de agregados na classe de 8,00 a 4,75 mm, foi mais elevada na RT1 em relação à CN1, apresentando valor médio de $66,01 \%$, refletindo em um maior DMG $(4,18 \mathrm{~mm})$. Esse resultado demonstra o potencial do pinus em manter a estabilidade dos agregados até mesmo nas camadas inferiores, tendo em vista que o DMG na camada seguinte $(0,20$ a $0,40 \mathrm{~m})$ não se diferiu do $\mathrm{CN} 1 \mathrm{e}$, inclusive, foi maior $(1,79 \mathrm{~mm})$.

Na RT2, camada de 0,00 a 0,05 m, os valores de distribuição do tamanho dos agregados formados

TABELA 4: Coeficientes de correlação de Spearman entre o diâmetro médio geométrico, porcentagem de agregados em cada classe e atributos físicos e químicos encontrados na segunda área de estudo.

TABLE 4: Spearman's correlation coefficients between the geometric mean diameter, percentage of aggregates in each class and physical and chemical attributes found in the second area of study.

\begin{tabular}{lcccccc}
\hline \multirow{2}{*}{ Atributo do solo } & \multirow{2}{*}{ DMG $(\mathrm{mm})$} & \multicolumn{5}{c}{ Classes de Agregados $(\mathrm{mm})$} \\
\cline { 3 - 7 } & & $8,00-4,75$ & $4,75-2,00$ & $2,00-1,00$ & $1,00-0,21$ & $<0,21$ \\
\hline Areia total $\left(\mathrm{g} \mathrm{kg}^{-1}\right)$ & $-0,06$ & $-0,06$ & $-0,02$ & 0,19 & 0,01 & $-0,02$ \\
Silte $\left(\mathrm{g} \mathrm{kg}^{-1}\right)$ & $0,56^{* *}$ & $0,57^{* *}$ & $0,36^{*}$ & $-0,28$ & $-0,55^{* *}$ & $-0,42^{*}$ \\
Argila $\left(\mathrm{g} \mathrm{kg}^{-1}\right)$ & $-0,35$ & $-0,38^{*}$ & $-0,26$ & 0,06 & $0,39^{*}$ & 0,30 \\
Densidade do solo $\left(\mathrm{Mg} \mathrm{m}^{-3}\right)$ & $-0,26$ & $-0,29$ & 0,04 & 0,32 & 0,20 & $-0,10$ \\
Porosidade total $\left(\mathrm{m}^{3} \mathrm{~m}^{-3}\right)$ & 0,13 & 0,13 & 0,00 & $-0,22$ & $-0,09$ & 0,18 \\
Grau de compactação $(\%)$ & $-0,28$ & $-0,31$ & 0,02 & 0,34 & 0,22 & $-0,09$ \\
Carbono orgânico total $\left(\mathrm{g} \mathrm{kg}^{-1}\right)$ & $0,58^{* *}$ & $0,64^{* *}$ & 0,12 & $-0,69^{* *}$ & $-0,48^{* *}$ & $-0,21$ \\
Al $\left(\mathrm{cmol} \mathrm{dm}^{-3}\right)$ & $-0,22$ & $-0,20$ & 0,01 & 0,24 & 0,10 & 0,21 \\
Ca $\left(\mathrm{cmol} \mathrm{dm}^{-3}\right)$ & 0,24 & 0,31 & $-0,13$ & $-0,38^{*}$ & $-0,12$ & $-0,20$ \\
Mg $\left(\mathrm{cmol} \mathrm{dm}^{-3}\right)$ & 0,18 & 0,26 & $-0,14$ & $-0,26$ & $-0,06$ & $-0,11$ \\
$\mathrm{~K}\left(\mathrm{mg} \mathrm{dm}^{-3}\right)$ & 0,32 & 0,35 & 0,09 & $-0,30$ & $-0,23$ & $-0,27$ \\
P $\left(\mathrm{mg} \mathrm{dm}^{-3}\right)$ & $0,53^{* *}$ & $0,60^{* *}$ & 0,03 & $-0,61^{* *}$ & $-0,43^{*}$ & $-0,25$ \\
pH em água & $-0,01$ & $-0,08$ & 0,03 & $-0,00$ & 0,09 & $-0,18$ \\
pH smp & 0,07 & 0,04 & $-0,03$ & $-0,10$ & 0,04 & $-0,15$ \\
CTC em pH do solo $\left(\mathrm{cmol} \mathrm{dm}^{-3}\right)$ & $-0,20$ & $-0,15$ & $-0,19$ & 0,07 & 0,15 & 0,27 \\
CTC em pH 7 (cmol $\left.\mathrm{dm}^{-3}\right)$ & $-0,06$ & 0,01 & $-0,09$ & $-0,01$ & $-0,02$ & 0,18 \\
\hline
\end{tabular}

Em que: $\mathrm{DMG}=$ diâmetro médio geométrico; $\mathrm{CTC}=$ capacidade de troca de cátions; * significativo a $5 \%$ de probabilidade; $* *$ significativo a $1 \%$. 
não diferiram estatisticamente do campo natural, o que pode estar relacionado ao fato de os teores de COT também não diferirem nessa camada, com 47,6 e 40,6 $\mathrm{g} \mathrm{kg}^{-1}$, respectivamente (Tabela 2). Entre os usos do solo, o DMG teve correlação altamente significativa com os teores de silte $(\rho=0,56)$, COT $(\rho=0,58)$ e P $(\rho$ $=0,53)$, conforme descrito na Tabela 4 . Em solos com elevada acidez, ricos em óxidos de $\mathrm{Fe}$ e Al, o fósforo da solução do solo tende a formar fosfato de ferro e de alumínio (altamente insolúveis), mantendo fixadas grandes proporções de fósforo total na superfície do solo (ABRÃO et al., 2015). Dessa forma, a adsorção de P aumenta as cargas negativas na superfície das partículas, favorecendo a CTC do solo e a formação de agregados.

Ressalva-se que a RT2, na camada de 0,00 a $0,05 \mathrm{~m}$, manteve percentuais de agregados estáveis semelhantes à sua área-testemunha. A partir dessa evidência é possível inferir que, nessa camada, o estoque de carbono orgânico foi parcialmente restabelecido, considerando que o povoamento na época da coleta se encontrava com 13 anos de idade. Dessa forma, a matéria orgânica incorporada à camada mineral superficial do solo tende a atingir quantidades em equilíbrio com as condições do ambiente, conforme se observa na área-testemunha; confirmando a relação existente entre o conteúdo de carbono e a idade do florestamento (BOCHNER et al., 2008; FACHINI, 2012). Chen e Shrestha (2012) perceberam baixa estabilidade de agregados logo após as práticas de corte raso e queima, no entanto, os efeitos foram sendo reduzidos ao longo do tempo, corroborando com Martín, Díaz-Raviña, Carballas (2012). Considera-se ainda, a importância da atuação das raízes de absorção nas camadas superficiais, que possuem elevada taxa de renovação e atuam ligando-se aos minerais do solo por meio da liberação de exsudatos orgânicos. Mesmo após a morte dessas raízes, a rede de hifas e fungos pode persistir no solo por alguns meses, devido à interação dessas raízes com os minerais no solo (TISDALL; OADES, 1982).

Analisando a camada de 0,05 a $0,20 \mathrm{~m}$, na classe de agregados de 8,00 a $4,75 \mathrm{~mm}$, verificou-se que o campo natural apresentou diferença significativa, possuindo maior valor médio para essa classe $(36,17 \%)$, mostrando-se altamente estável e, inclusive, na camada de 0,20 a $0,40 \mathrm{~m}(15,42 \%)$. Em contrapartida, a RT2 apresentou aumento da estabilidade de agregados com diâmetros menores. Observou-se ainda, que o CN2 apresentou maior teor de COT $\left(32,1 \mathrm{~g} \mathrm{~kg}^{-1}\right)$, diferindo significativamente da RT2 $\left(27,2 \mathrm{~g} \mathrm{~kg}^{-1}\right)$. Esse resultado possivelmente reflete o manejo adotado na área, o qual promoveu a redução no teor de material orgânico pela queima e corte raso do povoamento, expondo mais facilmente o solo às intempéries do ambiente. Nesse caso, o uso do fogo causa alterações físico-químicas no solo conforme a temperatura atingida durante esses eventos. Umas das principais consequências é a combustão da matéria orgânica, principal agente cimentante das partículas no solo. De acordo com os resultados, observou-se que na RT2 ocorreu uma redução dos macroagregados devido possivelmente ao enfraquecimento dos mecanismos biológicos de estabilização dos agregados a longo prazo, tendo em vista que, após perturbações dessa natureza, o solo necessitaria de um período maior para se reestruturar nas camadas mais subsuperficiais. Logo, a remoção da floresta, a queima dos resíduos vegetais e o excessivo revolvimento do solo causa diminuição do aporte e aumento da degradação da matéria orgânica, desestabilizando a estrutura do solo (ABRÃO et al., 2015); de modo que as partículas orgânicas fragmentam-se por meio da ação dos organismos do solo e pela decomposição microbiana, reduzindo a estabilidade dos macroagregados e aumentando a estabilidade de agregados menores.

Nas duas áreas de estudo avaliadas, os povoamentos de pinus e suas testemunhas tiveram redução do DMG de agregados estáveis em água em decorrência da diminuição dos teores de COT em profundidade, em consonância com os resultados encontrados em Wendling et al. (2005) e Morales et al. (2010). Tais valores estão relacionados à diminuição dos níveis de COT com o aumento da profundidade do solo, visto que em ambas as áreas a correlação foi altamente significativa entre os teores de COT e o DMG, sendo encontrados valores de $\rho=0,62$ e 0,58 , para a RT1 e RT2, respectivamente.

A matéria orgânica atua de forma direta e indireta no processo de agregação do solo, seja pelas suas características e propriedades intrínsecas ou pela alteração do meio (MORALES et al., 2010). Nesse sentido, somente o material orgânico sem transformação biológica não promove efeito na estrutura do solo. É necessária a contribuição de fungos e bactérias para a formação e estabilização intra e interagregados, por meio da excreção de polissacarídeos, formação de húmus, ou através do envolvimento das partículas primárias e agregados pelas hifas dos fungos (OADES, 1993). Wohlenberg et al. (2004) complementam afirmando que a própria matéria orgânica atua como fonte de energia para os micro-organismos no processo 
de agregação do solo. Por se tratar de um Cambissolo de caráter húmico, o solo apresenta elevados teores de material orgânico e carbono orgânico acumulado no horizonte superficial, fato relacionado às baixas temperaturas e elevada acidez do solo (baixo $\mathrm{pH}$ ), inibindo a atividade de micro-organismos decompositores. Além disso, as substâncias húmicas são altamente polimerizadas, permanecendo mais tempo no solo.

Considera-se ainda que a quantidade de material orgânico existente sobre o solo é consequência da massa aportada e de sua velocidade de decomposição. Desse modo, a composição da serapilheira depositada na superfície do solo, composta predominantemente por acículas, apresenta uma decomposição e incorporação mais lenta se comparada ao material orgânico fornecido pelas gramíneas (WIESMEIER et al., 2009). No entanto, a quantidade de raízes e exsudatos orgânicos provenientes dessas e também das acículas depositadas na superfície, favorecem a agregação do solo e sua estabilidade (KATO et al., 2010). Em trabalho envolvendo serapilheira de áreas com floresta secundária e com Mimosa caesalpiniaefolia, foram encontrados maiores teores de suberina e polifenóis nas mesmas, reduzindo a decomposição dos resíduos no solo e favorecendo o acúmulo de material orgânico, contribuindo com o aumento do DMP, DMG e índice de estabilidade de agregados (BOCHNER et al., 2008). Wohlenberg et al. (2004) também ressalvaram a importância da matéria orgânica como agente de agregação das partículas do solo, aumentando os indicadores de qualidade física do solo.

\section{CONCLUSÃO}

A estabilidade de agregados na camada superficial de até $0,05 \mathrm{~m}$ de um Cambissolo Húmico não é alterada com a mudança de uso de campo natural para povoamento com pinus com 13 anos de idade, bem como em área de segunda rotação, após 30 anos com florestamento.

Nas camadas mais profundas $(0,05$ a $0,20 \mathrm{~m}$ e 0,20 a $0,40 \mathrm{~m})$ da área de primeira rotação (com 13 anos), a estabilidade dos agregados maiores é mantida, ao passo que essa estabilidade não ocorre em área de segunda rotação, sugerindo que após a queima e o corte raso, as condições de equilíbrio dos mecanismos biológicos para a estabilização dos agregados ocorrem a longo prazo.

\section{REFERENCIAS}

ABRÃO, S. F. et al. Alterações químicas de um Cambissolo Húmico causadas pelo florestamento com Pinus taeda L. em área de campo natural. Floresta, Curitiba, v. 45, n. 3, p. 455-464, 2015.

BOCHNER, J. K. et al. Matéria orgânica e agregação de um planossolo sob diferentes coberturas florestais. Cerne, Lavras, v. 14, n. 1, p. 46-53, 2008.

BUCZKO, U.; BENS, O.; HÜTTL, R. F. Water infiltration and hydrophobicity in forest soils of a pine-beech transformation chronosequence. Journal of Hydrology, Amsterdam, v. 331, n. 3-4, p. 383-395, 2006.

CHEN, H. Y. H.; SHRESTHA, B. M. Stand age, fire and clearcutting affect soil organic carbon and aggregation of mineral soils in boreal forests. Soil Biology \& Biochemistry, Santa Barbara, v. 50, p. 149-157, 2012.

EMBRAPA. Centro Nacional de Pesquisa de Solos. Manual de Métodos de Análise de Solo. 2. ed. Rio de Janeiro: Embrapa Solos, 2011. 230 p.

EMBRAPA. Centro Nacional de Pesquisa de Solos. Sistema brasileiro de classificação de solos. 3. ed. Rio de Janeiro: Embrapa Solos, 2013. 353 p.

FACHINI, L. Frações e estoques de carbono orgânico em solo do planalto catarinense cultivado com pinus. 2012. 49 f. Dissertação (Mestrado em Manejo do Solo) - Universidade do Estado de Santa Catarina, Lages, 2012.

GUBIANI, P. I.; REINERT, D. J.; REICHERT, J. M. Método alternativo para a determinação da densidade de partículas do solo - exatidão, precisão e tempo de processamento. Ciência Rural, Santa Maria, v. 36, n. 2, p. 664-668, 2006.

INDÚSTRIA BRASILEIRA DE ÁRVORES. Relatório Anual 2016. São Paulo: IBÁ, 2016. 96 p.

KATO, E. et al. Propriedades físicas e teor de carbono orgânico de um latossolo vermelho-amarelo do cerrado, sob diferentes coberturas vegetais. Bioscience Journal, Uberlândia, v. 26, n. 5, p. 732-738, 2010. KEMPER, W. D.; CHEPIL, W. S. Size distribuition of aggregates. In: BLACK, C. A. Physical and 
mineralogical methods. Madison: ASA \& SSSA, 1965. p. 495-509.

MARTÍN, A.; DÍAZ-RAVIÑA, M.; CARBALLAS, T. Short- and medium-term evolution of soil properties in Atlantic forest ecosystems affected by wildfires. Land Degradation \& Development, Brighton, v. 23, n. 5, p. 427-439, 2012.

MARTINS, S. G. et al. Avaliação de atributos físicos de um latossolo vermelho distroférrico sob diferentes povoamentos florestais. Cerne, Lavras, v. 8, n. 1, p. 32-42, 2002.

MORALES, C. A. S. et al. Qualidade do solo e produtividade de Pinus taeda no planalto catarinense. Ciência Florestal, Santa Maria, v. 20, n. 4, p. 629-640, 2010.

OADES, J. M. The role of biology in formation, stabilization and degradation of soil structure. Geoderma, Amsterdam, v. 56, n. 1-4, p. 377-400, 1993.

REICHERT, J. M. et al. Reference bulk density and critical degree-of-compactness for no-till crop production in subtropical highly weathered soils. Soil \& Tillage Research, Amsterdam, v. 102, n. 2, p. 242-254, 2009. REICHERT, J. M.; REINERT, D. J.; BRAIDA, J. A. Qualidade dos solos e sustentabilidade de sistemas agrícolas. Ciência \& Ambiente, Santa Maria, v. 26, p. 26-48, 2003.

RESENDE, M. et al. Pedologia: base para distinção de ambientes. 6. ed. Viçosa: UFLA, 2014. 378 p.

SHRESTHA, B. M. et al. Soil aggregate- and particle- associated organic carbon under different land uses in Nepal. Soil Science Society of American Journal, Madison, v. 71, n. 4, p. 1194-1203, 2007.

TEDESCO, M. J. et al. Análise de solo, plantas e outros materiais. 2. ed. Porto Alegre: Departamento de Solos: UFRGS, 1995. $174 \mathrm{p}$.

TISDALL, J. M.; OADES, J. M. Organic matter and qater-stable aggregates in soils. European Journal of Soil Science, Oxford, v. 33, n. 2, p. 141-161, 1982.

VICENTE, T. F. S. et al. Relações de atributos do solo e estabilidade de agregados em canaviais com e sem vinhaça. Revista Brasileira de Engenharia Agrícola e Ambiental, Campina Grande, v. 16, n. 11, p. 1215-1222, 2012.

WENDLING, B. et al. Carbono orgânico e estabilidade de agregados de um Latossolo Vermelho sob diferentes manejos. Pesquisa Agropecuária Brasileira, Brasília, v. 40, n. 5, p. 487-494, 2005.

WENDLING, B. et al. Densidade, agregação e porosidade do solo em áreas de conversão do cerrado em floresta de pinus, pastagem e plantio direto. Bioscience Journal, Uberlândia, v. 28, supl 1, p. 256-265, 2012.

WIESMEIER, M. et al. Depletion of soil organic carbon and nitrogen under Pinus taeda plantations in Southern Brazilian grasslands (Campos). European Journal of Soil Science, Oxford, v. 60, n. 3, p. 311-487, 2009.

WOHLENBERG, E. V. et al. Dinâmica da agregação de um solo franco-arenoso em cinco sistemas de culturas em rotação e em sucessão. Revista Brasileira de Ciência do Solo, Viçosa, v. 28, n. 5, p. 891-900, 2004.

YODER, R. E. A direct method of aggregate analysis of soil and a study of the physical nature erosion losses. Journal American Society of Agriculture, Madison, v. 28, n. 5, p. 337-351, 1936. 